\title{
Outcome of Clubfeet by Physiotherapist Instructed Ponseti Method: A Case Study of 5 Years
}

\author{
Forhadul Hoque', Jobair Khan², Sanjana Zaman², Gias U Ahsan² and Mohammad Delwer Hossain \\ Hawlader $^{2 *}$
}

${ }^{1}$ LMRF Healthcare, Chittagong, Bangladesh

${ }^{2}$ Department of Public Health, North South University (NSU), Dhaka, Bangladesh

"Corresponding author: Hawlader MDH, Assistant Professor, Department of Public Health, North South University, Dhaka, Bangladesh, Tel: +88 01747439378; E-mail: mohammad.hawlader@ northsouth.edu

Received: December 15, 2018; Accepted: December 22, 2018; Published: December 26, 2018

\begin{abstract}
Ponseti Method is a gold standard technique for the treatment of clubfoot or congenital Talepas Equino Varus with the proven higher success rate in physiotherapy practice. Aim of the study was to find out the evidence that application of Ponseti method bring complete clinical and functional outcome. A baby boy at the age of 20 days attended a health care center (LMRF) without history of any complication either himself or mother. We diagnosed him as bilateral typical clubfeet and score of his mid foot were higher compare to the hind foot throughout the casting period. Total Pirani score was 5.5 in both feet at the day of first casting. It required total 8 casting, where 1.5 and 1 were the Pirani score of the right and left foot respectively. He received manipulation (exercise therapy) just prior to the casting. Pirani score became 0 after tenotomy. Patient received the brace with the 6 size after 21 days of tenotomy. Patient also did the intense exercise therapy and highly encouraged to engage in gaming. Total 13 visits were needed up to the age of 5 years. Shoe size was changed over the time and at the last visit size was 13 . Treatment was ended with the performance of all functional movement accurately with stability of the clinical outcome. This study can conclude that application of Ponseti method bring complete clinical and functional outcome and not only casting but also complete maintaining phase under physio brought the good result of club foot.
\end{abstract}

Keywords: Clubfoot; Ponseti method; Pirani score; Clinical and functional outcome

\section{Introduction}

Clubfoot, also known as Congenital Talipes Equino Varus (CTEV), is a complex structural misalignments and congenital deformity of the foot usually involve soft tissue and bony structures in the hindfoot, midfoot and forefoot [1]. Though there is no universally accepted classification exists [2] but some other classified into a) positional clubfoot, b) idiopathic clubfoot, and c) secondary clubfoot [1]. Over the last three decades, Ponseti technique applied as most practiced treatment with

Citation: Hawlader MDH, Khan J, Zaman S, et al. Outcome of Clubfeet by Physiotherapist Instructed Ponseti Method: A Case Study of 5 Years. Clin Case Rep Open Access. 2018;1(2):115. 
www.yumedtext.com | December-2018

outstanding consequences [1]. Application of Ponseti method in idiopathic clubfoot, casting in correction phase conducted by physiotherapist group were found more effective with fewer recurrence compare to the directed by surgeon group [3,4].

\subsection{History}

A male baby referred by a physician and brought by his mother at 20 days of age in a health care center (LMRF), Chittagong, Bangladesh. His mother has history of normal pregnancy with the total duration of 9 months without suffering from any complication neither mother nor baby, even not during the delivery process. No other child was noted with clubfoot in the family.

\subsection{Evaluation}

Clinically, baby was found to have bilateral foot involvement, symptom of rigidity, and diagnosed as a typical CTEV by the physiotherapist at the age of 20 days. No other deformity in hip, knee or spine was noted.

\subsection{Outcome measure}

Outcome was measured by two ways: a) Clinical outcome: was measured by whether appearance of any relapse event or not [5] by using Pirani Score (PS), b) Functional outcome: was measured by well-functioning foot with good mobility [6].

\subsection{Treatment}

The treatment started in first phase with gradual correction of the deformity: cavus, midfoot inversion, heel varus and the rigid equinus need to be corrected. Pirani scoring was done prior to each casting, considering age and severity of the condition followed by tenotomy. In second stage, correction was maintained by three-month intense use of brace and continued up to the age of five [7,8]. It also comprised with the a) Therapeutic Exercise: One of the key arsenal what parent has to accomplish three times a day and applied according to the demand of the condition. The exercises were i) Calf muscle stretching, PROM- dorsi flexion muscle: sustain 10 seconds with 10 repetitions ii) Full squatting during toileting. Instruction was provided to play at least one to one and half hour every day. Encouraged to running, rope jumping, cycling, stair climbing, heel walking and toe walking. b) Counseling: Started before the onset of treatment, during casting, bracing and after bracing [7].

\subsection{Tenotomy}

The trained and designated surgeon performed a successful percutaneous Achilles tenotomy (pAT) with local anesthesia. Before the tenotomy, at $8^{\text {th }}$ visit scoring reduction was continuing, PS became 0.5 at mid feet (both) but 1.5 , 1 (right and left respectively) was at hind foot. Patient's feet were treated with three consecutive serial casts over the three weeks after the tenotomy.

\subsection{Bracing}

Patient visited once in every sixth month and followed the following guidelines: a) from the date of bracing start to 3 months: It kept open for 20 min in every 8 hrs, 3 times a day. b) 3 months to 3 years: 12 hours during night and 3-4 hrs during day time c) 3 years to 5 years: $10-12$ hrs only at night. 
www.yumedtext.com | December-2018

\subsection{Bracing Records}

Regular visit was continued every six months interval up to the 5 years of age. It took total 13 visits until the feet were large enough to fit into the smallest available boots. Before every follow up, planter reflex was tested for confirming sensory function of the feet. Throughout the bracing period, PS maintained 0, initially 6 size shoes were given and boots were well fited (TABLE 1). Patients visited the health center for consecutive two weeks for the fitting of the foot. Patient was instructed to wear the brace for $23 \mathrm{hrs}$ but brace off not more than 1 hour. Patient was strongly advised to avoid walking over the first three months. Shoe size was 6 at first four visit, from there shoe size changed in every second visit size up to the $10^{\text {th }}$ visit and last three visits he could use the 13 size. At the sixth visit, patient started to walk freely and instructed for running and squatting as much as possible. From the $8^{\text {th }}$ visit to the age of 2 years, he went through, allowed and instructed for the vigorous activities and exercises like jumping, rope jumping, heel walking, toe walking and cycling as per considering his ability.

TABLE 1. Regular followup checklist of the bracing period.

\begin{tabular}{|c|c|c|c|c|}
\hline Date & Boots Appointment & Pirani Score & Shoe Size & Comments \\
\hline 12-05-11 & $\begin{array}{c}\text { Initial Fitting of } \\
\text { Boot }\end{array}$ & 0 & 6 & Good \\
\hline 26-05-11 & 2 weeks check & 0 & 6 & Good \\
\hline 04-07-11 & $1^{1 / 2}$ month check & 0 & 6 & Good \\
\hline 22-08-11 & 3 months check & 0 & 6 & Good \\
\hline 28-11-11 & 6 months check & 0 & 8 & Good \\
\hline $30-05-12$ & 1 year check & 0 & 8 & Good \\
\hline 04-11-12 & $11 / 2$ years check & 0 & 11 & Good \\
\hline 05-12-12 & 2 years check & 0 & 11 & Good \\
\hline 05-06-13 & $21 / 2$ years check & 0 & 12 & Good \\
\hline 01-01-14 & 3 years check & 0 & 12 & Good \\
\hline 02-07-14 & 4 years check & 0 & 13 & Good \\
\hline 13-05-15 & $41 / 2$ years check & 0 & 13 & Good \\
\hline 16-09-15 & 5 years check & $\mathbf{0}$ & 13 & End of treatment \\
\hline
\end{tabular}

\section{Result}

Bar chart shows that total 8 castings were required and scoring was reduced gradually. Casting interval was checked and changed once in every week. Pirani score was measured before each casting. Casting performed after positioning and 
manipulation of the foot. It was observed that, after the first casting, PS of the medial crease of the corrected feet was 0 . During $4^{\text {th }}$ casting, PS of right foot found to be 0 but left one was 0.5 which continued to the next casting and developed 0 from the $6^{\text {th }}$ casting. At $8^{\text {th }}$ casting, PS of taller head coverage seen 0 in both feet also total PS of mid foot reached to 0 . In observing hind feet, only PS was found 0 in left leg at $5^{\text {th }}$ casting. Rigid equinous constantly maintain PS as 1 at $4^{\text {th }}$ casting and maintained 0.5 from $5^{\text {th }}$ casting to the end. Empty heel also observed 0.5 PS from $2^{\text {nd }}$ to $8^{\text {th }}$ casting. At the last casing, in both midfoot, all 3 signs became disappeared in comparing hind feet, PS remain 1.5 in left and 1 in right (FIG. 1-3).

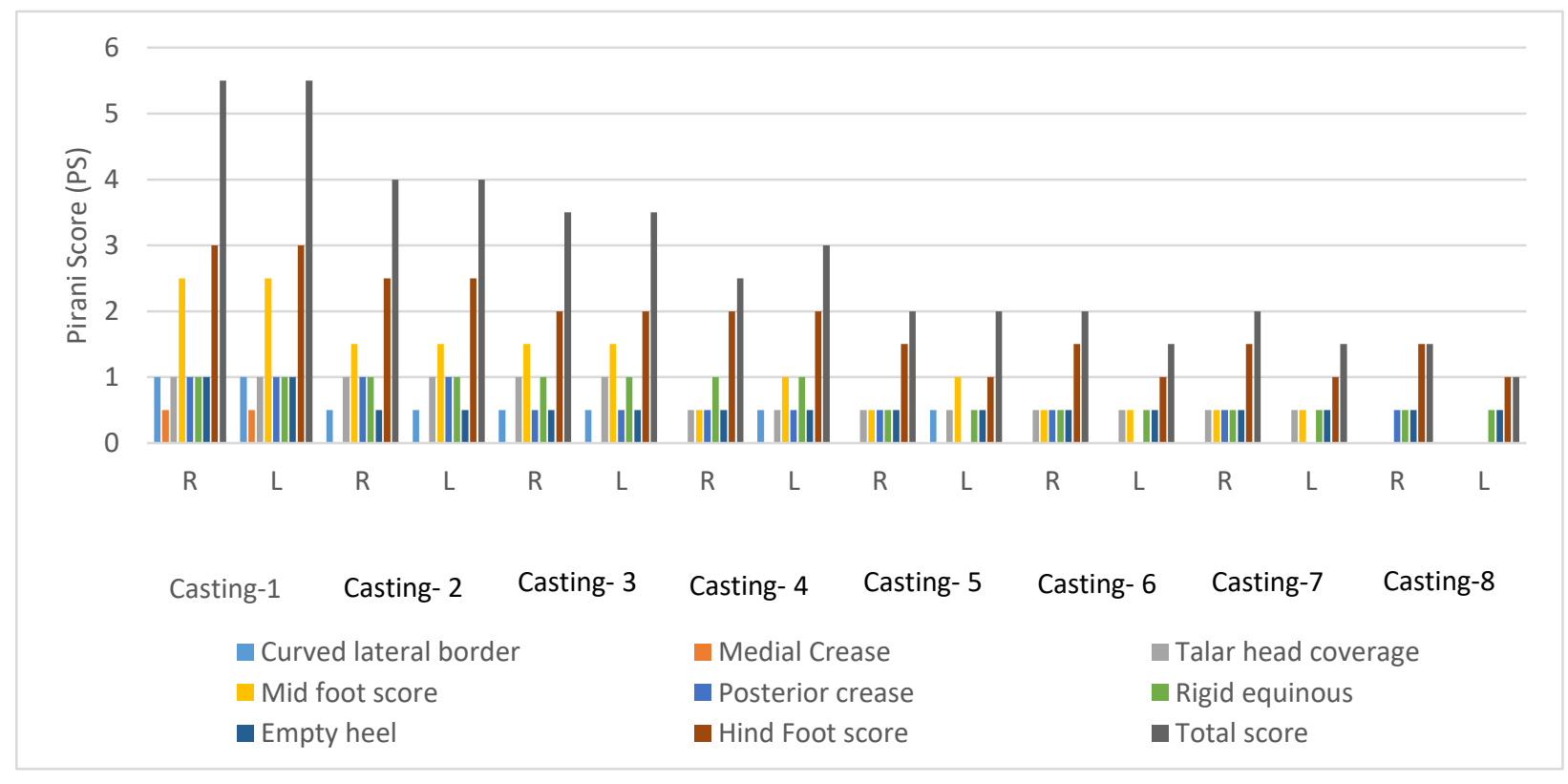

BAR CHART. Pirani score of the foot during first phase of treatment.

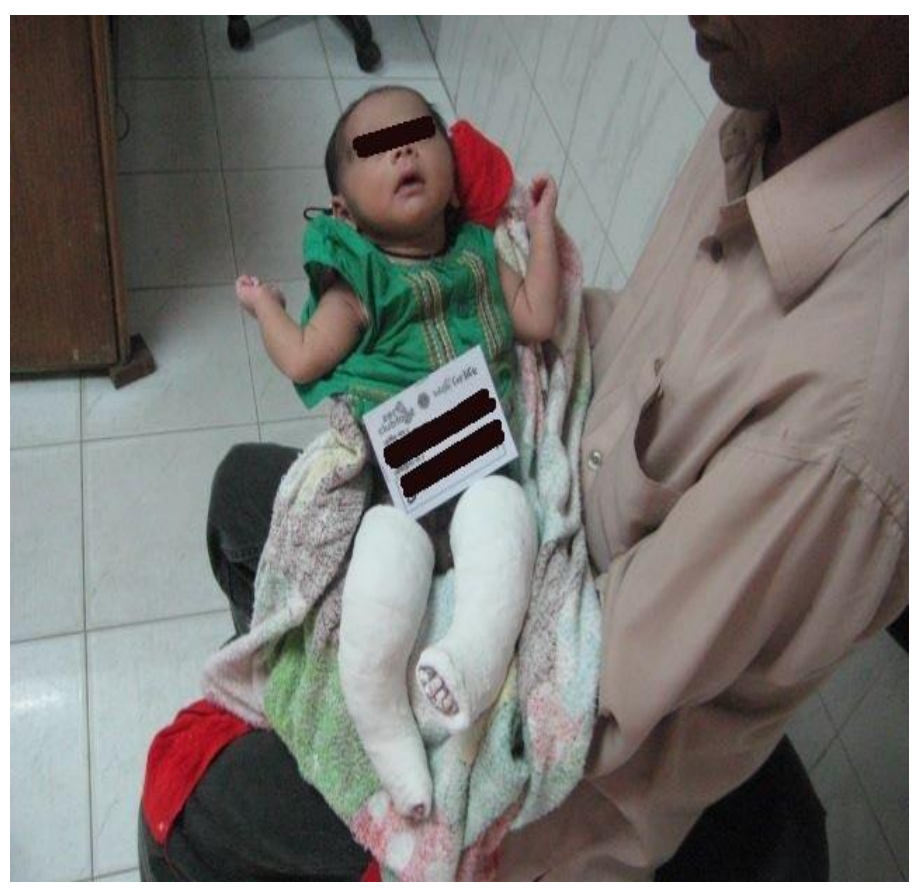

FIG. 1. During casting period. 


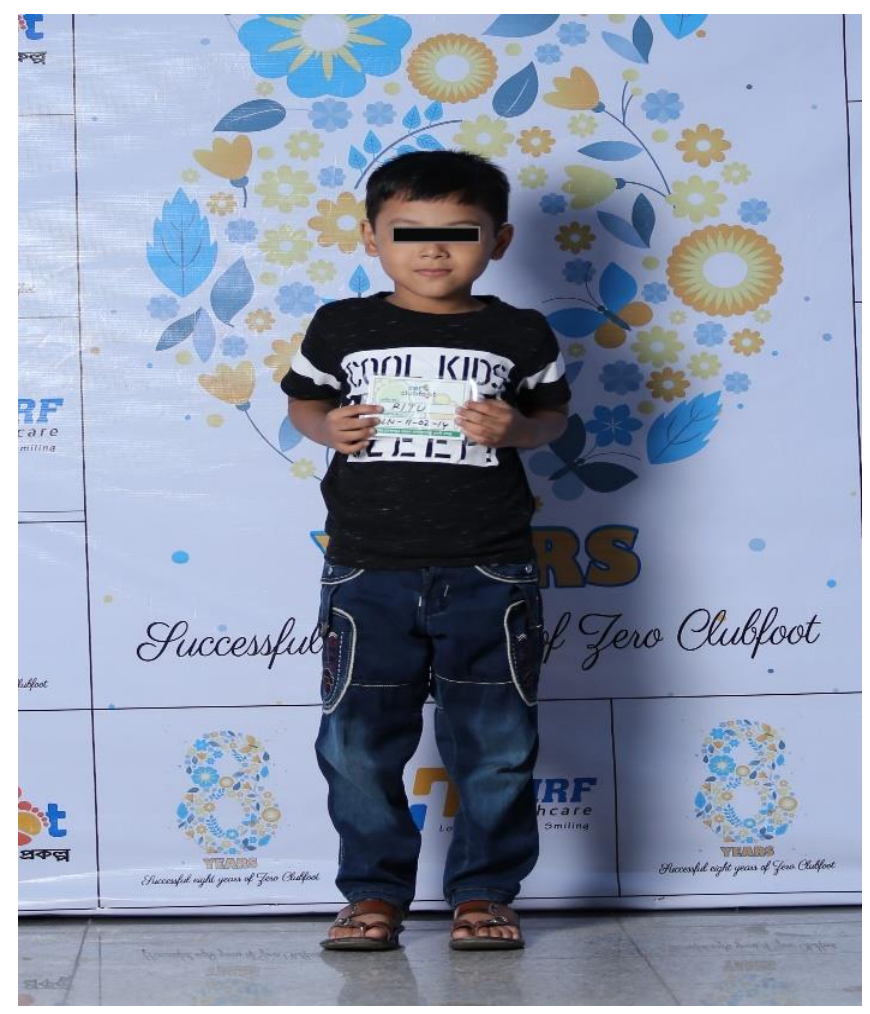

FIG. 2. After treatment.

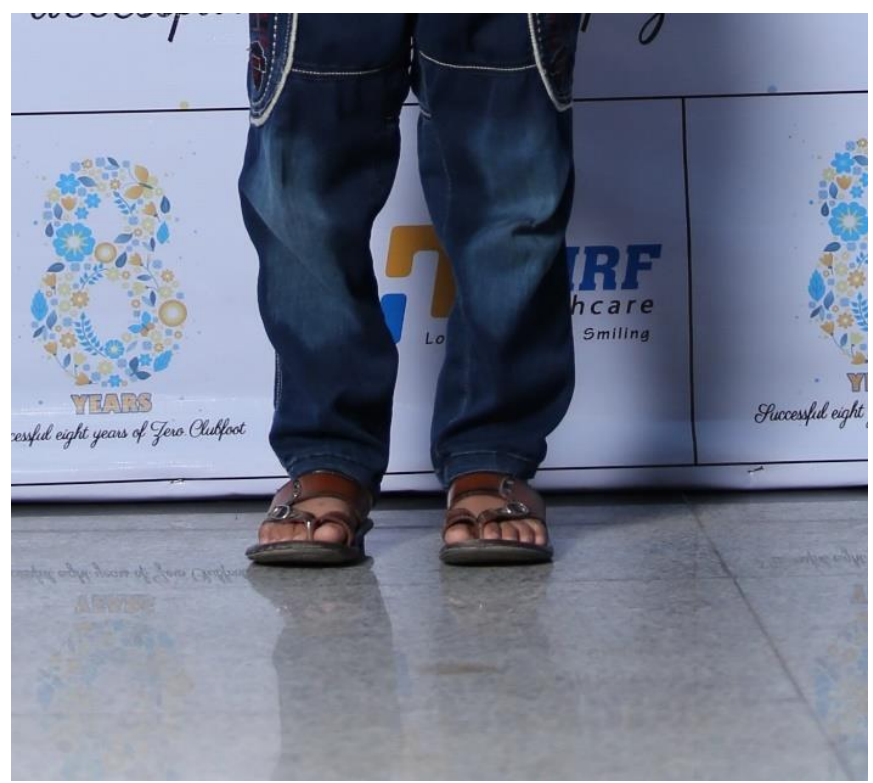

FIG 3. Completely normal feet after treatment.

\section{Discussion}

The baby was diagnosed as typical clubfeet at the age of 20 days where feet were involved bilaterally with rigidity. In recent work suggest that $97.7 \%$ children were diagnosed within their age of 5 years, $10.5 \%$ parents informed with a family history of CTEV [9]. It observed in single foot or bilateral feet but with 50\% of cases being both and affected less common in female than male with noted range of ratios 1.6:1 to $3: 1$ [1]. Study also found, it is usually appeared immediately after birth and 
www.yumedtext.com | December-2018

usually developed in between 9 to 14 weeks gestation [10]. Under a trained and skilled physiotherapist (Ponseti practitioner), patient was continued and completed correction, and maintenance phase. Patient visited and changed casting once in every week. It required total 8 visits for the above knee casting. Cast was removed by the attendee at the day of visit in the corridor of the center. Similarly in other work, physiotherapist performed manipulation and casting and cast removing and bracing [11]. Previous study supported our procedure regarding removal and changing of casting which is once in a week [5] but their average number of casting sessions was lower (5.5) than us. Maximum success was noted above knee casting compare to the below knee failure rate was $37.5 \%$ and significantly required longer treatment times [8].

At the last visit of corrective phase, PS were 1.5 in right and 1 in left foot. In other research, after completion of the corrective phase PS of 1 or less achieved in $85.0 \%$ cases, of which 37 feet was a PS of 0; 99 achieved 0.5 ; and 73 was PS of 1. In addition, $11.0 \%(n=27)$ feet was PS of 1.5 and only $4.0 \%(n=10)$ unable to achieve score of 1.5 or less [9]. pAT tenotomy with local anesthesia done by the surgeon at the aim of release equinus. Previous study shown that up to $90 \%$ of cases require an Achilles tenotomy to correct remaining equinus deformity [5]. Other work also demanded tenotomy indicated by clinical assessment thereafter surgeon or medical officer conducted pAT tenotomy with local anesthesia [11].

Patient received Steenbeek Foot Abduction Brace (SFAB) for the shoe size of 6 and during the first three month patient was not allowed to off it from the feet more than an hour. He used the brace until the end of treatment around the age of 5 years with the shoe size of 13. Considering clinical outcome was remain stable as PS was 0. Ponseti himself depicted in his first case series, the use of the foot abduction orthosis after 3 months of full time bracing for an additional mean duration of 21 months (ranging 10-30 months) with a recurrence noted in 56\% of cases [9]. Relapse took place in nine studies, which was due to the non-adherence of bracing regime and other factors such as low income and social economic status [12].

In our case, clinically no sign of relapse returned and functionally able to lead independent work. Clinical outcome was measured weather arisen of any sign of the CTEV. To ensure the neurological function, reflex of the planter flexion checked regularly and found intact. Functional outcome was measured by the perfect walking, running, jumping and squatting of the patient. Other work reported about the functional outcome where in case of $88 \%$ did not have any limitation, $7 \%$ quoted occasional limitation and $3 \%$ noted ongoing limitation [11].

\section{Conclusion}

From our study, we can conclude that not only casting period but also complete maintaining phase under physio brought the good result of club foot if we can continuously counsel, instruct and teach therapeutic exercise to the parents. Even though before the tenotomy in clinical assessment by PS was 1.5 in right and 1 in left but after the tenotomy it become 0 and never relapse until the end of the treatment. In functional assessment, patient was able to do all activity as normal child as perform.

\section{Recommendation}

Modern technology may apply to measure foot alignment and function. Still it is not clear about long term functional capability after completion of treatment, it means weather functional state throughout the life remain same or improved or worsen. Therefore, follow up study need to be continued until later life. 
www.yumedtext.com | December-2018

\section{Ethical Consideration}

Permission for this study was taken from the ethical committee of the North South University and LMRF Healthcare. Informed written consent was obtained from the patient of the child before and during the procedures.

\section{Funding}

This research was supported by LMRF Healthcare.

\section{Acknowledgement}

We would like to thanks Dr. Mr. Shamim Khan, Managing Trustee and CEO of LMRF Healthcare for his continuous support and allowing us to conduct the research in LMRF Healthcare. We are also thankful to the study participant and his parents for keeping their patience for such a long time.

\section{REFERENCES}

1. O'Reilly N, Muzenda KR, Lowe R, Thomas., E. and Maeseele, T. Introduction to Clubfoot. https://www.physiopedia.com/Introduction to_Clubfoot; Retrieved on 18/02/18.

2. Kaustubh MC, Gaurav MM, Gaurang MP, et al. Early results of clubfoot management by Ponseti method. Int J Orthop Sci. 2017;3(3):460-3.

3. Janicki JA, Narayanan UG, Harvey BJ, et al. Comparison of surgeon and physiotherapist-directed Ponseti treatment of idiopathic clubfoot. J Bone Joint Surg Am. 2009;91(5):1101-8.

4. Dobbs MB, Morcuende JA, Gurnett CA, et al. Treatment of Idiopathic Clubfoot: an historical review. Iowa Orthop J. 2000;20:59-64.

5. Loof E, Andriesse H, André M, et al. Gait in 5-year-old children with idiopathic clubfoot: A cohort study of 59 children, focusing on foot involvement and the contralateral foot. Acta Orthop. 2016;87(5):522-8.

6. Lohan I. Treatment of Congenital Clubfoot Using The Ponseti Method Workshop Manual. 2nd edn. Seattle, WA: Global Health. Health Education Using Low Cost Publications, USA; 2011.

7. Mejabi JO, Esan O, Adegbehingbeb OO, et al. A prospective cohort study on comparison of early outcome of classical Ponseti and modified Ponseti post tenotomy in clubfoot management. Ann Med Surg (Lond). 2017;24:347.

8. Radler C. The Ponseti method for the treatment of congenital club foot: review of the current literature and treatment recommendations. Int Orthop. 2013;37(9):1747-53.

9. Malagelada F, Mayet S, Firth G, et al. The impact of the Ponseti treatment method on parents and caregivers of children with clubfoot: a comparison of two urban populations in Europe and Africa. J Child Orthop. 2016;10(2):101-7.

10. Smythe T, Chandramohan D, Bruce J, et al. Results of clubfoot treatment after manipulation and casting using the Ponseti method: experience in Harare, Zimbabwe. Trop Med Int Health. 2016;21(10):1311-8.

11. Gray K, Pacey V, Gibbons P, et al. Interventions for congenital talipes equinovarus (clubfoot). Cochrane Database of Systematic Reviews. Cochrane Database Syst Rev. 2012;18(4):CD008602.

12. Ganesan B, Luximon A, Al-Jumaily A, et al. Ponseti method in the management of clubfoot under 2 years of age: A systematic review. PLoS One. 2017;12(6):e0178299. 